\title{
The superior germplasm of goat and buffalo.
}

\author{
Osama Ibrahim Azawi* \\ Department of Surgery and Theriogenology, College of Veterinary Medicine, University of Mosul, Iraq
}

Accepted on October 24, 2017

\section{Editorial}

Goat and buffalo husbandry are becoming a lucrative enterprise in the developing counters. The Shami breed can produce half milk of bovines characterized by a long reddish-colored coat with long ears and a Roman nose with various subtypes ranging from North America through the Middle East into Asia. The Shami goat has received special attention by farmers, by virtue of its early maturity, higher milk production, meat and high percentage of twins and triplets, in comparison to other breeds. The river buffalo produce high milk with more than $11 \%$ fat (Bubalus bubalis), which can be found from India and further west to Egypt and some parts of Europe. The Mediterranean buffalo of the river. The buffalo, compared with all other domestic animals produce high quality of milk, which is characterized with $7 \%$ fat and more than $4 \%$ protein. This type of buffalo is primarily used for milk, meat and skin production, with 50 diploid numbers of chromosomes. Goat and buffalo are a photoperiodic species to be considered a "short day" species. They have heats throughout the year but tend to result more fertile when day light hours decrease. This characteristic is due to their tropical origins. They came from North equatorial areas where the availability of forage coincides with the period in which the dark hours increase. Therefore, it has supposed, that animals which part urate in the most suitable period for survival of the offspring, were selected. It seems that they kept this characteristic even when they were transferred to places where forage is always available. The worldwide seek and need for milk, meat and food encourages researchers in research centres. universities and united nation organizations for extensive work to improve production and reproduction for goat and buffalo population. These species of animals need an improvement of their germplasm and genetic selection. Artificial Insemination of using selected Shami buck semen is the easiest method to be used to achieve intensification of goat production. To start, it is imperative to develop a suitable semen diluent to preserve Shami goat semen at $5^{\circ} \mathrm{C}$, which is an important stage of equilibration before freezing of the diluted buck semen. Protocols for goat semen dilution and preservation continue to be developed due to the wide range of results found for sperm motility, considered the parameter of choice to determine the degree of sperm damage inflicted by the diluents' containing materials and procedures. In order to lower the metabolism of spermatozoa and prolong storage, semen is generally cooled following dilution. The diluents normally contain egg yolk, to avoid spermatozoa from cold shock, which occurs when sperm are subjected to temperatures lower than $15^{\circ} \mathrm{C}$. There is strong evidence to suggest that buck seminal plasma contains an enzyme called egg coagulating enzyme secreted in large quantities by the bulbourethral gland of the male goat, which triggered with the lecithins of egg yolk or milk-based media hydrolyses to lysolecithins and fatty acids. This hydrolysis produces a toxic substance lethal to spermatozoa. Thus this process is not possible to cool goat semen without a washing procedure removing the seminal plasma. This includes an initial dilution with a diluent free for egg yolk followed by centrifugation and discharge of the supernatant, before the semen is re-diluted with a diluent containing egg yolk. Such a practice is harmful to sperm and lowered the viability of spermatozoa. Another limiting factor in semen preservation is its exposure to the light during manipulation before storage, leading to formation of reactive oxygen species (ROS) with damage to sperm cell motility and genomic integrity. In buffalo, estrus detection as well as training buffalo bull for semen collection as some breeds are wild or semi wild. These problems must be paid attention by research centres and universities to resolve.

\section{*Correspondence to:}

Osama Ibrahim Azawi

Department of Surgery and Theriogenology

College of Veterinary Medicine

University of Mosul

Mosul

Iraq 